\title{
The Charge Structures of Aether and Substance
}

\section{Georgy Nikolskiy}

Russian State Scientific Centre for Robotics and Technical Cybernetics (RTC), St. Petersburg, Russia

Email: ngeorgii68@gmail.com

How to cite this paper: Nikolskiy, G. (2018) The Charge Structures of Aether and Substance. Open Access Library Journal, 5: e4379. https://doi.org/10.4236/oalib.1104379

Received: January 26, 2018

Accepted: February 10, 2018

Published: February 13, 2018

Copyright $\odot 2018$ by author and Open Access Library Inc.

This work is licensed under the Creative Commons Attribution International License (CC BY 4.0).

http://creativecommons.org/licenses/by/4.0/

\begin{abstract}
The hypothesis of the charge structures matter serves as the basis for defining the inner energies of "rest" an electron: $E_{e}=\alpha c /\left(2 \pi R_{e}\right)\left[\mathrm{s}^{-1}\right]$; a neutrino: $E_{n}=E_{e} \alpha^{3}$; a proton: $E_{p}=E_{e}\left(\alpha^{-2}-3 \alpha^{-1}\right) / 10$. "Relict" radiation is regarded as the wave background is caused by charges oscillations of the aether. Charge pair known as a neutrino is the elementary structure of the aether and participant of the energy and charges exchange reactions between the ethereal and inert structures. The internal spin dynamics of the charge structures of particles creates their inertness. The electron mass of "rest" is defined as the time density on its shell that forms the space-time unity: $M_{e}=\alpha /\left(2 \pi R_{e} c\right) \quad\left[\mathrm{s} / \mathrm{m}^{2}\right]$. Factors of randomness and periodicity in the radioactivity phenomenon are explained. The ethereal concept of the antiparticles origin is proposed.
\end{abstract}

\section{Subject Areas}

Classical Physics, Particle Physics

\section{Keywords}

Aether, Charge Structure, Electron, Neutrino, The Energy of "Rest"

\section{Introduction to the Philosophy of Objective Dualism}

The classical physics stands on the ideological concept of objectivity of nature existing independently of natural sciences. Modern quantum theory uses mainly the concepts of classical physics but subjectively in theory, it endows the object with a dual nature. The subjective dualism gives priority to the subject who develops the theory describing the object behaviour, arbitrarily. The subjective dualism logic rejects the objectivity of the Universe deductible from the "Big Bang" theory.

The objective dualism sees the reason of materiality in the objective phenomena of the interaction of the two polarities of electric charges, connected into 
structures that exist in two forms of motion. There is nothing in the world except electric charges, but the single loose charge can't be imagined. The electric forces fasten the elementary charges in structures objectively. The electron inertness or the mass of "rest" is a consequence of a stable process of charge structure rotation. The structural concept allows understanding then electron is bonded, answering the question Richard Feynman [1].

The subjective dualism of wave-corpuscular theory mixes the objective entities. The physics of objective dualism provides an understanding of the material nature of the aether, which is used to be the base of natural sciences before beginning of the 20th century [2] [3]. The subjective formalism of a relativity theory abolished the space ether whose place was taken by a physical vacuum. "The word 'aether' has gained a very negative connotation in the theoretical physics due to its previous association with the opposition to the relativity theory. We do not speak about aether, because it's a taboo" [4]. Ignoring of the fact of existence of the physical environment transferring light results in need to prove existence not only aether, but also the Universe that it obviously goes beyond competence of a mathematical formalism. The physicists who ostracised the notion of aether (A. Einstein, N. Bohr etc.), attempted to explain the nature of the "physical vacuum" [5]. Suspecting that the theory is incomplete, David Bohm thought that particles "similar to electrons" constitute the reality at sub-quantum level [6]. Bohm described the hypothetical field with a quantum potential distributed on all space. The hypothesis of the quantum potential worked in a continuous medium to explain the field connection and behavior of the parts. The matter did not reach to radical revision of the theory and consideration of the environment as structures from discrete elementary electric charges- "similar to electrons".

Many philosophers and physicists who understood that the "mainstream" course taken by physics was wrong wrote that "radical revision of relativity and quantum theories are necessary not somehow adapting present theories, but radically revising their logical and philosophical base" [7]. "One could now put forward solid arguments in favour of postulating aether" [8]. "Einstein's relativity theory does no longer explain all the problems regarding elementary particles and we should look for new ways of development" [9].

\section{The Electron Structure}

The electron is considered the carrier of the only elementary charge. A possibility of its existence as the stable, free particle having the energy of "rest" it is impossible to explain in the absence of the structure keeping the single electric charge [1]. The explanation can be found, if to considerate an electron as the charge structure in which the structural balance of forces is maintained. The electron is fastened by the interaction energy positive and two negative charges, representing dynamic structure-the spinning "top". The forces stay in balance if the charges are placed into the vertices of an equilateral triangle [10]. The energy potential of which the "resting" electron has: $E_{e}$, it is caused by dynamics 
of charge structure rotation with the light speed at which the internal energy and a moment-an electron spin save.

According to the classical representation the radius of a "structureless" electron is:

$$
R_{e}=2.82 \times 10^{-15} \mathrm{~m} ;
$$

the mass and the charge of an electron are smeared inside a volume with internal radius: $R_{c h g} \leq 10^{-17} \mathrm{~m}$. In alternative model the value: $R_{c h g}$ characterizes the radius of the only elementary charge. The internal energy of an electron is the quantum value determined as the rotation frequency of the charge structure:

$$
v_{e}=\alpha c /\left(2 \pi R_{e}\right)=1.23 \times 10^{20} \mathrm{~s}^{-1},
$$

where $\alpha=1 / 137$-the structure constant; $c$-the light speed.

Fundamental universal constant: $\alpha$ is known as the fine structure constant. It also universal characterizes the ratio of energies (frequencies) when compared the structural interaction of charges, manifested in two forms: rotational and vibrational [11].

Plank constant $\left(h=4.14 \times 10^{-15} \mathrm{eV} \cdot \mathrm{s}\right)$ allows defining the energy in the usual units:

$$
E_{e}=h v_{e}=0.511 \mathrm{MeV} .
$$

The structure acquires inertness and external freedom when the charges interaction energy is enough to start the electron spinning. The inertia or mass of the "rest" is the external manifestation of the internal energy of charges interaction inside the structure. Due to the internal dynamics the electron becomes an inert particle characterised by three degrees of freedom [11]. As a particle it participates in the construction of the atomic structures. Free mobile electron has external kinetic energy. Free movement and possession of the inert mass are a consequence of internal process of charge structure rotation. Internal dynamics of charges interaction creates the external inert electron mass of "rest", bond to the energy of "rest" by the known formula:

$$
M_{e}=v_{e} / c^{2}=\alpha /\left(2 \pi R_{e} c\right)=1370 \mathrm{~s} / \mathrm{m}^{2}
$$

Multiplying $M_{e}$ by Plank constant: $h=6.626 \times 10^{-31} \mathrm{~g} \cdot \mathrm{m}^{2} / \mathrm{s}$ - a coefficient for quantum units recalculation-we get the inert "rest" mass in the usual units: $M_{e M}=h M_{e}=9.1 \times 10^{-28} \mathrm{~g}$.

We can get Formula (1), characterizing the rotation of the charges structure, as the ratio of turnaround time: $T_{e}=2 \pi R_{e} / c$ to the area a thin disk, localizing the process of rotation: $S=2 \pi R_{e}^{2}$, multiplied by the transition constant: $\alpha / 2 \pi$ [12]. Electron is a "trap" which catches the electric charges when they come too close. Describing the stationary process of charge structure rotation with the speed of light we define the time density on the electron shell. The mass of "rest" of an electron is the quantum of inertness generated by electric forces. We define $M_{e}$ as the space-time unity or time density on the electron shell. By replacing the right part in Formula (1) with a constant: $10 / \alpha=1370$, we can estimate the speed of the electron shell pulsations: 


$$
c_{e}=R_{e} c=\alpha^{2} / 20 \pi=0.85 \times 10^{-6} \mathrm{~m}^{2} / \mathrm{s} .
$$

\section{The Charge Structures of Aether}

The fundament of the structural hierarchy of the material world is the energy and the information potential the aether. It is neither emptiness nor a physical vacuum but a continuous network made of discrete elementary electric charges that carries the light and also is the source of the background radiation called relict. In the aether network every charge is exposed to the forces of attraction and repulsion front and back, right and left, their action being balanced. The aether charges form a cubic structure similar to that of the silver halide photo emulsion, the difference being that the lattice nodes contain electric charges instead ions.

Neutrino is an elementary structure consisting of a pair of charges that participates in energy and charges exchange reactions between inertial matter structures and the aether. Neutrinos are not registered practically as a free particles flow with the energy received at birth. However, we know the value of the neutrino oscillations energy maximum: $E_{n} \leq 0.28 \mathrm{eV}$ [13], which characterises the process of charges interaction in the ether structure. The charge pair or neutrino does not possess freedom in the ether structure. The relation between the "rest" energy of the electron possessing three levels of freedom and of the neutrino which has no freedom within ether structure is expressed through the structural constant: $E_{n} \alpha^{0}=E_{e} \alpha^{3}$ [12]. The ether concept allows to estimate the "rest" energy of the unfree neutrino: $E_{n}=E_{e} \alpha^{3}=0.2 \mathrm{eV}$. This estimation complies with the known experiment data [13]. The energy of "rest" an neutrino is the oscillation energy of charges at frequency: $v_{n}=E_{n} / h=4.83 \times 10^{13} \mathrm{~s}^{-1}$ that should include the wave and the inertial components. Each charge in the ether network interacts, with highest priority, with the six nearest charges. The charge pairs, as well as single charges in the ether structures are linked together with electric forces. In the general network a neutrino loses individuality. We can ascribe the estimated neutrino oscillations energy maximum to the energy of charge oscillations around the middle balanced position: $E_{c h g}$. The meaning of the concept and the value of energy do not depend on the direction of oscillating charges movement. This is why to estimate the energy density of the ether matter we sum the module values: $q_{c h}=\sum_{i}\left|E_{\text {chgi }}\right| / V$, where $V$-volume.

The ether background radiation is generated by acceleration and deceleration of the oscillating charges. In the cosmic ether the spectre of the background or "relict" radiation comes to maximum at frequency: $v_{c}=1.6 \times 10^{11} \mathrm{~s}^{-1}$ $\left(h v_{c}=6.6 \times 10^{-4} \mathrm{eV}\right)$. The wave energy density of the cosmic background radiation is also known to be: $q_{c r}=0.25 \mathrm{eV} / \mathrm{cm}^{3}$ [14]. These estimations relate to the rare "atmosphere" of the cosmic ether. The known experimental data allows to judge about the wave component of oscillations charges energy. Question about the neutrino full energy and mass of "rest" remains unresolved. Neutrino is not a photon with zero mass or a particle with the internal energy of rest. Therefore, we interpret the energy of the background radiation of the aether, as a 
part of the total energy of the oscillating charges. We suppose that the full energy of interaction in the pair of charges $\left(E_{c n}\right)$ divides in equal parts into wave and inertia components. In this case the full energy of a space neutrino equals double of the wave component energy: $E_{c n}=1.32 \times 10^{-3} \mathrm{eV}$. The sum energy density of the inertial and the wave components is: $q_{c}=0.5 \mathrm{eV} / \mathrm{cm}^{3}$. This data helps to estimate the charge density: $q_{c h g} \approx 380 \mathrm{~cm}^{-3}$ and the average distance between the charges in space: $L_{c} \approx\left(q_{c h g}\right)^{-1 / 3} \approx 1.38 \mathrm{~mm}$. The energy charge density of the ether "atmosphere" of the substance is higher than the density of the cosmic ether in proportion that corresponds to the charges interaction energies. The relation of the energies of "rest" neutrino in Earth and space conditions is: $E_{n} / E_{c n} \approx 150$.

The charge oscillations energy manifests itself in wave and inertial forms. Up to present time an experiment verification of the ether inert properties has not been possible. The recent information about registration of the gravitation waves can be interpreted as the discovery of the inertial component of the ether charge structures oscillations. The charge concept opens up the real prospect of creating a unifying theory of physical forces. We can assume that gravity is a consequence of surface tension forces ethereal network at the interface with moving bodies and particles.

\section{Proton and Neutron}

Particles of substance acquire external inertia, thanks to the inner intensive dynamics of charges interaction-to rotation of structure. The lightest inert structure of an electron consists of three charges. A charge structure consisting of seven charges also maintains a balance of the interaction forces and a transition to the high level of energy. The hexagon of six charges rotating around a positive central charge is a structural model of a proton. The key role of the proton in creating material charge structures shows that the proton possesses additional levels of freedom as compared to the electron. It is easy to show that the "rest" energies of the proton and the electron are related by a simple formula that reflects the inner processes of charges interactions within their structures [12]. We can get the values of proton "rest" energy \& mass:

$$
E_{p}=E_{e}\left(\alpha^{-2}-3 \alpha^{-1}\right) / 10, v_{p}=2.26 \times 10^{23} \mathrm{~s}^{-1}, M_{p}=2.51 \times 10^{6} \mathrm{~s} / \mathrm{m}^{2} .
$$

Within the charges concept, being considered the electric forces form the inner energy potential of "rest" of the proton, neutron and nuclides with the high charge density. The experimental data and the vocabulary of the modern physics can provide us a fresh vision to the genius predictions of Greek philosopher Plato. His wonderful hypothesis reflects the intuitive understanding of the matter being composed of geometric shapes: triangle-the electron, hexagon-the proton, cube-the neutron. These structures make up all the diverse forms of the substance we can observe. Plato conceived what we call aether as a "mother", "nursing" substrate that is not homogeneous and interacts with the structures of polyhedrons. Plato's hypothesis inspired Werner Heizenberg when he has devel- 
oped the quantum theory. He thought that "one can hardly move forward in the modern atomic physics without knowledge in Greek philosophy" [9].

The priority "strong" form of the interaction energy is made up rotation that does not exclude influence on the nuclide stability of the "weak" oscillation form liming the lifetime of structure. The dynamic stability of the charge nuclear structure depends on the balance of the electric interaction forces. The unstable neutron structure consisting of eight charges should have a cube shape that can balance the attraction and repulsion forces. The known data [15] confirms this idea. The dynamic instability of the neutron structure is explained by lack of a permanent centre or axis of rotation. The free neutron is known to decay, relatively fast, into a proton, an electron and an antineutrino. The "rest" energy of the neutron that exceeds by $1.3 \mathrm{MeV}$ the internal energy of the proton procures the energy balance of the reaction. The emergence of the antineutrino means that two charges undergo a transition from the aether into the electron structure to keep the charge balance.

The weak interaction theory that tried to explain the neutron decay has found irregularities of the time invariance of the neutron-antineutron oscillations-a phenomenon which arises from subjectivity of the wave-corpuscle dualism that sees no principal difference between the inert particles and the aether waves. The neutron structure theory has received the Nobel Prize in physics allowing us to know the following: the neutron consists of a heavy core bearing $35 \%$ of the "elementary" positive charge and two virtual shells, one of them carrying 50\% of the negative electric charge and the other $15 \%$ of the positive charge [16]. This theory has allowed splitting an elementary, i.e. indivisible charge on parts, basing on the speculative interpretation of experimental data.

\section{The Nuclide and Radionuclide Structures}

Protons and neutrons are regarded as the construction material for the atomic nuclei, in their structure they lose the individuality and are called nucleons. In the theory of the charge structures of matter there is no reason to describe the internal nuclear field by special physic forces qualitatively different from the electric forces. The nuclides structures made up from elementary electric charges that were parts of nucleons, and their total amount defines the nuclide mass. The nuclide gets seven charges from each proton and the neutrons give to the nuclide eight charges. The energy and the mass of the nuclide depend on the density and on the total number of the charges. The average charge density in the nuclei varies insignificantly within the limits set by the values of the charge density in the proton and neutron structures, in the range of: $(0.9-1.025) \times 10^{45} \mathrm{chg} / \mathrm{m}^{3}$. The estimations are made under the assumption that the nuclides shell is spherical with the following radius: $R_{N}=1.23 \times 10^{-15} A^{1 / 3}$, where $A$ stands for atomic weight. The nuclei charges structures are linked by the electric forces starting the structures rotation process. The quantum theory explains the dynamics of stable nuclear structures formation by "pairing effect" [17], that makes the charge 
structure take the position where the total angular momentum $J=0$ and the energy bracing together the charge structure reaches its maximum. The angular momentum of unstable nuclei: $J \neq 0$ takes the minimum value ensuring the structure lifetime. The "pairing effect" leading to rotation momentum minimization manifests itself as a "strong" interaction having the electric nature.

Into the inner nuclear energy makes the contribution the "weak" oscillations influencing on stability of a nuclide, causing in the unstable positions breaking the bonds that fasten nuclear structure. It must be note that nuclide stability is a relatively rare phenomenon. Today we know 300 stable nuclides and more than 3000 unstable radionuclides with charge structure that does not provide a perfect balance of the interaction forces. In this case the dynamics of the substructures rotation cycles in radionuclides with a certain period leads to critical states when the structure stability depends from weak oscillations. This hypothesis qualitatively explains the factors of randomness and cyclicity that characterize the processes of radionuclide decay. Also note that the existing theories of strong and weak interactions do not provide a qualitative explanation of internal nuclear processes that lead to decay of the unstable charge structures and do not offer a method estimation of the half decay period.

The superfluous charge density in the nucleus is considered a factor of radionuclide instability. The density limit that divides the stable and the unstable nuclei lies within of interval:

$(0.965-0.97) \times 10^{45} \mathrm{chg} / \mathrm{m}^{3}$. The lower limit was estimated using the charge density of the most important long-livers radionuclide of natural origin: ${ }_{19} \mathrm{~K}^{40}$, with half decay period $1.25 \times 10^{9}$ years.

The nucleus of ${ }_{19} \mathrm{~K}^{40}$ includes 301 charge (21 neutrons, each of them possessing 8 charges and 19 protons, containing 7 charges each). Out of them 160 charges are positive and 141 are negative. The total positive charge of the radionuclide is predicated by the difference. The cycle processes going on in the nuclei ${ }_{19} \mathrm{~K}^{40}$ put the radionuclide to a critical state when "weak" random oscillations break the internal nuclear links.

\section{The Ether Origin of Antiparticles}

The electron is formed of the aether charges if their oscillation frequency exceeds value: $v_{r}=2.5 \times 10^{20} \mathrm{~s}^{-1}$. The potential of the charges interaction energy reaches the level when the oscillation energy transforms into the internal energy of charge structure rotation. The six charges of the ether structure form two spinning tops with opposite rotation momentums: an electron and a positron. The positron birth helps to observe the save principles of charges, energy and momentum. In presence of the matter the positron annihilates together with an electron when the energy is being emitted in the shape of two photons. The charge structures concept gives a basis for ethereal interpretation of the antiparticles origin. Positron and antineutrino are short-lived excited states consisting of three and two aether charges respectively, induced by the transition of charges 
from aether into substance. To calculate the reaction charge balance we sum the charges converted from the substance structures into aether with a plus and those, that are converted the reverse way-from aether into substance-with a minus. The principle of charges save in the reactions is supported by following examples: free neutron ( 8 charges) decays into a proton (7), an electron (3) and an antineutrino (-2): $8=7+3-2$; in proton-proton reaction where a deuteron (15) a neutrino (2) and a positron ( -3 ) are created, the total being: $7+7=15+2-$ 3.

The radionuclide ${ }_{19} \mathrm{~K}^{40}$ beta-decay with probability $89 \%$ resulting in the production of a stable nucleus ${ }_{20} \mathrm{Ca}^{40}$, an electron and an antineutrino. We calculate the charges number of the reaction products and see that it corresponds to the original quantity of charges in the nucleus ${ }_{19} \mathrm{~K}^{40}(301): 300+3-2=301$, where 300 is the number of charges in the nucleus ${ }_{20} \mathrm{Ca}^{40}$; 3-number of electron charges; -2-number of the antineutrino charges. The second reaction, its probability being about $11 \%$, is an electron capture which results ${ }_{18} \mathrm{Ar}^{40}$ (302), a positron and a neutrino. The charge balance of the alternative reaction: $302-$ $3+2=301$ also fits, because the emergence of an antiparticle (positron) means that three charges leave aether; the neutrino supplies two charges to aether. In this last reaction the significant difference in the energies connecting the structures before and after the reaction is carried to aether as an electromagnetic radiation with frequency: $v_{r}=3.5 \times 10^{20} \mathrm{~s}^{-1}$.

\section{Conclusions}

The hypothesis of the charge structures of aether and substance served as the basis that helped to reconsider of the physical ideology. The thin matter of aether consists of the elementary electric charges. The charge pairs-neutrinos are considered as participants of the exchange transition processes in the reactions where energy and charges transform between aether and the inert structures. The neutrino possesses a zero level of freedom and the energy of "rest" from the charges oscillations in aether. The charges oscillations energy manifests in the inertial form and as the aether background radiation.

The electron "rest" energy and mass are result from the rotation of the charge structure consisting of two negative charges and one positive. The idea of the freedom levels of the elementary charge structures serves as the basis for establishing the relations between the "rest" energies of neutrino, electron and proton. The inertness is an external manifestation of rotation processes induced by the inner interaction of the electric charges. The total inertness is determined by the total action of the internal and external forces in the structures hierarchy. The electron mass of "rest" is defined in the quantum units: $M_{e}=10 / \alpha \mathrm{s} / \mathrm{m}^{2}$.

Both the strong and the weak intranuclear forces are reduced to a common electric type. The "strong" interaction is caused by the "pairing effect" of the charge substructures rotation in the nuclides. The instable radionuclides go to critical states periodically, when the structure stability depends on the weak oscillations 
influence. This hypothesis explains qualitatively the factors of randomness and periodicity of the radioactivity phenomenon.

The charge structures concept offers a basis for explanation of the antiparticles origin validated by checking of the save charges law in the exchange reactions between aether and particles.

\section{References}

[1] Feynman, R. (1989) The Feynman Lectures on Physics. Vol. 5, Chapter 1, Electromagnetism. Science, Moscow.

[2] Vavilov, S. (1987) Isaak Newton, Chapter VI. Nauka, Moscow.

[3] Whittaker, E. (2001) A History of the Theories of Aether and Electricity. Regular and Chaotic Dynamics, London.

[4] Laughlin Robert, B. (2005) A Different Universe: Reinventing Physics from the Bottom Down. Basic Books, New York, 120-121.

[5] Einstein, A. (1966) On the Method of Theoretical Physics. Collected Papers v. IV, Moscow.

[6] Bohm, D. (1987) Hidden Variables and the Implicate Order, in Quantum Implications. Routledge \& Kegan Paul, London, 38.

[7] Bernal, J. (1956) Science in History, Moscow. The MIT Press, Cambridge.

[8] Dirac, P. (1951) The Evolution of the Physicist's Picture of Nature. Nature, 5, 166.

[9] Heisenberg, W. (1990) Physics and Philosophy. Part and Whole. Nauka, Moscow.

[10] Nikolskiy, G. (2015) Third Element. From Aether Structure to the Matter Construction. LAP LAMBERT, Saarbruken.

[11] Nikolskiy, G. (2015) Aether and Time. Heretical physics. Everything Wrong. LAP LAMBERT, Saarbrücken.

[12] Nikolskiy, G. (2015) We Cannot Live without Cosmic Internet. What the Dark and the Light Matter Are Woven from. Holography of the Soul. LAP LAMBERT, Saarbrücken.

[13] Thomas, S.A., Abdalla, F.B. and Lahav, O. (2010) Upper Bound of $0.28 \mathrm{eV}$ on Neutrino Masses from the Largest Photometric Redshift Survey. Physical Review Letters, 105, 031301.

[14] Zeldovich, Y. and Novikov, I. (1975) Structure and Evolution of the Universe. Nauka, Moscow.

[15] Llanes-Estrada, F.J. and Navarro, G.M. (2011) Cubic Neutrons. Modern Physics Letters A, 27, 1250033. https://doi.org/10.1142/S0217732312500332

[16] Schelkin, K. (1965) Virtual Processes and Nucleon Structure. Physics of the Macroworld. Atomizdat, Moscow.

[17] Valantin, L. (1986) Subatomic Physics: Nuclei and Particles. Further Development. Vol. 2. Mir, Moscow. 